\title{
Seeing measurement on Sasahuine mountain, Moquegua, Perú
}

\author{
C. Ferradas-Alva ${ }^{1}$, G. Ferrero ${ }^{1}$, M. Huamán ${ }^{1}$, W. Guevara-Day ${ }^{1}$, \\ E. Meza ${ }^{1}$, J. Samanes ${ }^{1}$, and P. Becerra ${ }^{1}$ \\ ${ }^{1}$ Departamento de Astrofísica, Agencia Espacial del Perú, CONIDA, Perú
}

\begin{abstract}
One of the greatest factors that significantly affect the quality of astronomical images is the atmospheric turbulence causing what we call "seeing". We present results of the reduction and photometry of astronomical images obtained at the Sasahuine mountain astronomical site (4511 m.a.s.l.), located in the Southern Peruvian Andes, in the department of Moquegua, near the town of Cambrune. These data show preliminary seeing measurements for this site. The present work is part of a bigger investigation program called JANAX which seeks to evaluate potential astronomical observation sites in Peruvian territory through a series of observation missions. The program's aim is to gather data to validate the site for the future construction of a National Astronomical Observatory. The observations were made using an SBIG ST-7MX CCD camera and a BVR standard filter set, attached to a MEADE LX200 356mm telescope.
\end{abstract}

Keywords. site testing, techniques: photometric

\section{Introduction}

If there were not a terrestrial atmosphere nor interstellar dust between an external source of radiation (eg. a celestial body) and our telescope (on Earth), this radiation would reach the telescope and form a diffraction pattern called Airy disk, due only to the optical effects on the telescope (because of the light diffracting and producing a pattern with concentric dark and bright rings around the image of the object). However, this is not the case. In fact, radiation coming from a star traverses various obstacles, thus affecting its optical path in different ways. If we neglect the effects produced by interstellar material (which is a good approximation) the greatest obstacle between stellar radiation and our telescope is the terrestrial atmosphere. "Seeing" is the astronomical term for the extent of resolution degradation of an image caused by the Earth's atmospheric turbulence. This degradation in image quality results from fluctuations in the refractive index of air as a function of position and time above the site. Seeing is often the limiting factor in the quality of astronomical observations at a given site, and in Astronomy it is quantified using the stellar profile, measuring the full width at half maximum (FWHM) which is the angular size of the image of a star with half the peak intensity level. CONIDA conducted a search for the best potential astronomical sites in the Peruvian territory based on meteorological information from 40 years of data from the International Satellite LandSurface Climatology Project (ISLSCP), the Surface meteorology and Solar Energy (SSE) database and continously monitoring of several meteorological stations. To accomplish this search, CONIDA created a program denominated JANAX. This program looked for sites over 4000 meters of altitude with the lowest cloud coverage (cf. Barrios 2007), humidity and wind velocity, preferably far from active volcanoes, the lowest geological risk and favourable logistic situations (i. e. roads, water and energy proximity). It was found that the Andes of Moquegua, in Southern Peru, had the best conditions within 


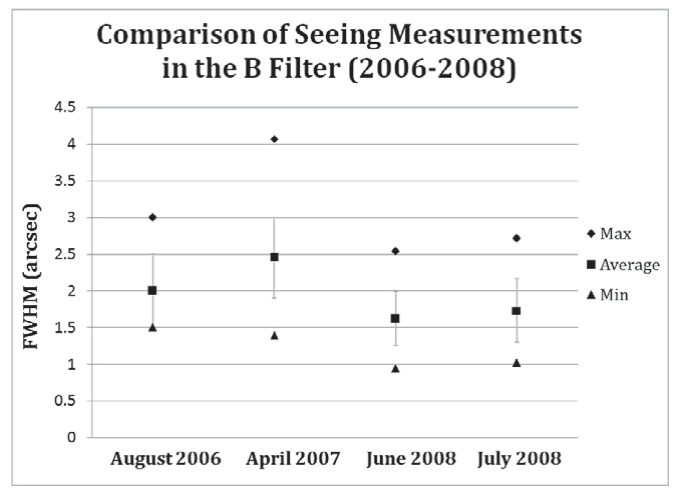

(a) B Filter

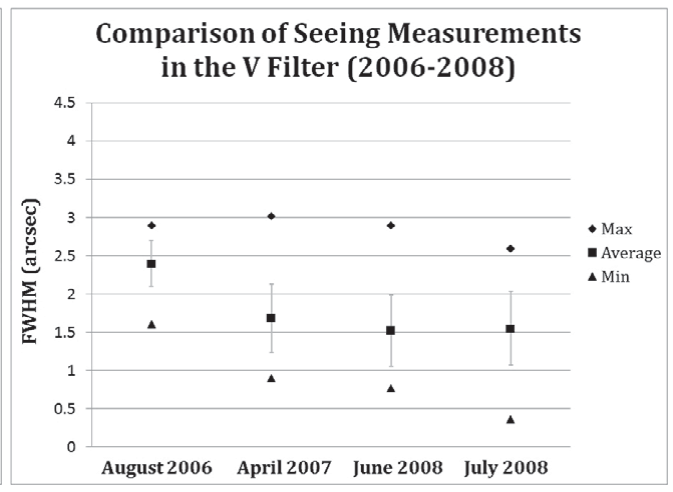

(b) V Filter

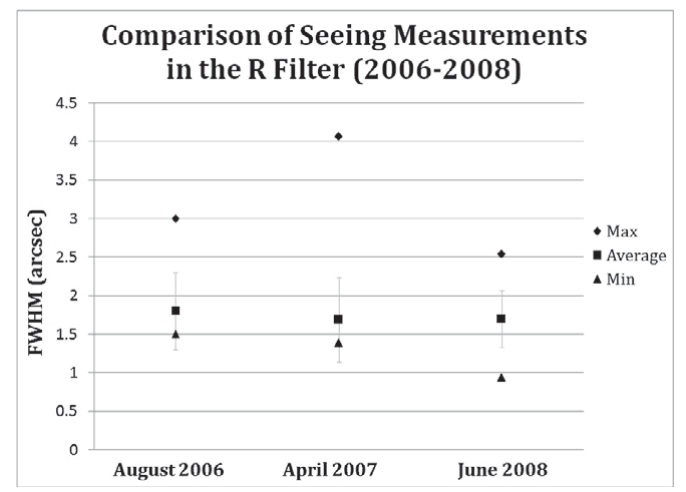

(c) R Filter

Figure 1. Comparison of seeing results for the JANAX missions from 2006 through 2008. Each image shows the average (squares), maximum (diamonds) and minimum (triangles) values.

the country (Ferrero et al. 2005; 2006) and, after a visual sites evaluation, measurements of seeing and extinction coefficients were started in Sasahuine mountain.

\section{Instruments and Observations}

Based on this initial search and as part of the JANAX program, CONIDA decided to send astronomical observation missions to the Sasahuine mountain site, in order to evaluate the quality of the sky there. JANAX, spanned a period of 4 years from 2004 through 2008, was carried out in ten observation missions. These missions were achieved during different times of the year. Digital images were taken for either a single star or a star open cluster during one or two nights of observation per mission. Seeing measurements were made during new moon nights every few minutes during 2 to 3 hours per night, providing that cloud coverage allowed imaging.

An ST7-MX SBIG CCD camera was used, with a BVR standard filter set. This system was attached to a Meade 356 mm/ 14 inch LX200 telescope with Ritchey-Chretien optical design. 


\section{Data Processing}

The processing and reduction of the images was done using the IRAF† (Image Reduction and Analysis Facility) environment in both Unix and Linux operating systems (Coulman et al. 1986). This process is necessary because the images need to be corrected for instrumental effects that contaminate the data. The first steps were the mandatory image corrections from bias, dark frames and flat fields. Next, aperture photometry and transformation to the standard magnitude system of Johnson \& Morgan were performed (Rolhfs 1949) on each of the stars imaged (single stars such as Achernar ( $\alpha$ Eri) or open clusters such as the Jewel box cluster (NGC 4755) and the Butterfly cluster (M6)), using the IRAF phot task. The output from this task provided us with instrumental magnitudes and FWHM values for each star, in each image, of each filter. Finally, the airmass was calculated, using the IRAF astcalc task. In order to obtain the final seeing measurements in seconds of arc, the airmass for each star in each image in each filter was needed. To find it, the IRAF astcalc task was used, with a script whose job was to read an input table containing the necessary parameters (image name, star coordinates, date and time of observation and exposure time), and calculate the airmass based on these parameters. Once the airmass was calculated, the final seeing value for each star was calculated according to the usual formula, which accounts for airmass correction and pixel scale:

$$
\text { SEEING }=0.49 \times\left[F W H M /\left(\text { airmass }^{0.6}\right)\right]
$$

Where FWHM is the full width at half maximum value obtained from the radprof task, and $0.49 \mathrm{arcsec} / \mathrm{pix}$ is the pixel scale measured from the same images.

\section{Seeing Results and Conclusions}

Figure 1 shows the results of the average seeing values obtained for the visual, blue, and red filters at the Sasahuine mountain astronomical site. We compare the results of the different JANAX missions.

From the first mission in August of 2006 until the last one in July of 2008, we have observed a quantitative improvement in the results, probably due to the placement of a structure to keep the telescope's tracking system warmer so that it could work better. We did this because the extreme cold weather made the tracking system work with difficulty. For the $\mathrm{V}$ filter, the highest average seeing value was 2.4 arcsec, corresponding to JANAX 2006, and the minimum value obtained was 0.4 arcsec, corresponding to JANAX July 2008. Even though the highest average value is 2.4 arcsec, a simple comparison of these results with other seeing analysis, like the one done for the Huayao Observatory in Huancayo (Peru) by Pereyra and Baella (2003) demonstrate that Sasahuine mountain has enviable seeing. The average seeing value for all filters is 1.8 arcsec, which is a very good result, considering the type of equipment used. We would expect this result to improve with the use of better equipment. If more observations and seeing calculations confirm the values obtained in the present work, we will be provided with an excellent argument to obtain funding for the construction of a professional astronomical observatory at this site.

$\dagger$ IRAF is distributed by the National Optical Astronomy Observatories, which are operated by the Association of Universities for Research in Astronomy, Inc., under cooperative agreement with the National Science Foundation. 


\section{Acknowledgements}

The authors thank the entire staff of the Astrophysics department at CONIDA for their support and guidance throughout the whole image reduction process. We also thank the National Meteorology and Hydrology Service for their support in the logistics of the observation missions.

\section{References}

Barrios, E., et al. 2007, Análisis de Cobertura Nubosa en el Sur del Territorio Peruano Utilizando Imágenes GOES-12 en las Bandas 03 y 04, CONIDA, internal report.

Coulman, C. E., et al. 1986, The Observation, Calculation, and Possible Forecasting of Astronomical Seeing. Publications of the Astronomical Society of the Pacific 98, 376-387.

Ferrero, G. et al. 2005, Búsqueda de Sitios Astronómicos en el Perú: Resultados de la Expedición Janax I, ECIPERU 2005, ISSN: 1813-0194, vol. 2, n. 1, 17, available at http://www.cienciaperu.org/images/revista/vol2n1.swf

Ferrero, G. et al. 2006, Análisis de Variables Determinantes en la Búsqueda de Sitios Astronómicos en el Perú, ECIPERU 2006, ISSN: 1813-0194, vol. 3, n. 1, 25, available at http://www . encuentrocientificointernacional.org/revista/Vol3N1.pdf

NASA, Goddard Space Flight Center, International Satellite Land-Surface Climatology Project (ISLSCP) available at http://www.gewex.org/islscp.html

NASA, Surface Meteorology and Solar Energy available at http://eosweb.larc.nasa.gov/sse/ Pereyra, A. \& Baella, N. 2003, Medidas de seeing en el Observatorio de Huancayo, REVCIUNI, Vol 7, n. 1, 103.

Rohlfs, K. et al. 1949, Lichtelektrische Dreifarben-Photometrie von NGC 6405 (M6), Zeitschrift für Astrophysik, Bd. 47, S. 15-23. 